\title{
FACTORES PROTECTORES DE LA DEPRESIÓN EN UNA MUESTRA DE ADULTOS MAYORES EN PUERTO RICO: AUTOEFICACIA, ESCOLARIDAD Y OTRAS VARIABLES SOCIO-DEMOGRÁFICAS
}

\author{
José A. Serra Taylor ${ }^{1 *}$, Carol Y. Irizarry-Robles ${ }^{1}$ \\ ${ }^{1}$ Universidad de Puerto Rico, Recinto de Río Piedras, San Juan, Puerto Rico
}

Recibido, diciembre 20/2013

Concepto de evaluación, noviembre 28/2014

Aceptado, diciembre 30/2014
Referencia: Serra-Taylor, J., \& Irizarry-Robles, C. Y. (2015). Factores protectores de la depresión en una muestra de adultos mayores en Puerto Rico: autoeficacia, escolaridad y otras variables sociodemográficas. Acta Colombiana de Psicología, 18(1), 125-134. DOI: 10.14718/ACP.2015.18.1.12

Resumen

El objetivo de esta investigación descriptiva-correlacional fue evaluar la relación entre autoeficacia, escolaridad y síntomas depresivos para identificar factores protectores de la depresión en una muestra de personas de edad avanzada de la población de Puerto Rico. Además, se examinaron otras variables sociodemográficas como el estado civil, con quién residen y el sexo, en relación con la autoeficacia y los síntomas depresivos. Se seleccionaron por disponibilidad 45 participantes con edades entre 60 y 86 años ( $M=69$; $\mathrm{DE}=7.37)$. Los instrumentos utilizados fueron la Escala Autoevaluativa de la Depresión de Zung (1965) y la Escala de Autoeficacia General de Baessler y Schwarcer (1996). La hipótesis planteó que, a mayor autoeficacia y escolaridad, menor sería el nivel de los síntomas depresivos en una muestra de adultos mayores de Puerto Rico. Los análisis estadísticos incluyeron pruebas t y los Coeficientes de Correlación de Pearson y de Regresión Múltiple. Se encontraron diferencias estadísticamente significativas para la autoeficacia y la escolaridad entre quienes no presentaban depresión y los que presentaban depresión moderada o severa. Además, se encontraron mayores niveles de depresión y menores niveles de autoeficacia en quienes habían enviudado. Los resultados sugieren que altos niveles de escolaridad y autoeficacia son factores protectores de la depresión en los adultos mayores, mientras que la viudez es un factor de riesgo para la depresión.

Palabras clave: depresión, autoeficacia, escolaridad, adultos mayores.

\section{PROTECTIVE FACTORS FOR DEPRESSION IN AN ELDERLY SAMPLE OF PUERTO RICO: SELF-EFFICACY, EDUCATIONAL LEVEL AND OTHER SOCIO-DEMOGRAPHIC VARIABLES}

\begin{abstract}
The aim of this descriptive-correlational study was to assess the relationship between self-efficacy, educational level and depressive symptoms in order to identify protective factors for depression in a sample of elderly people in Puerto Rico. Other socio-demographic variables such as marital status, living arrangements, and sex were examined in relation to self-efficacy and depressive symptoms. The selected sample consisted of 45 participants ranging in age from 60 to 86 years old (M=69; $\mathrm{SD}=7.37$ ). Measures employed included the Zung Self-Rating Depression Scale (1965) and the Baessler and Schwarcer's General Self-Efficacy Scale (1996). The hypothesis stated that the level of depressive symptoms would be lower when there is higher self-efficacy and higher educational level in a sample of Puerto Rican elderly people. Statistical analysis included $\mathrm{t}$ tests, the Pearson Correlation Coefficient and Multiple Regression Coefficient. Statistically significant differences for selfefficacy and educational level were found between those who did not show depressive symptoms and those with moderate to severe depression. In addition, higher levels of depression and lower levels of self-efficacy were found in those who had widowed. Results suggest that high levels of education and self-efficacy are protective factors for depression in elderly people, while widowhood is a risk factor for depression.

Key words: depression, self-efficacy, educational level, elderly
\end{abstract}

* Departamento de Consejería para el Desarrollo Estudiantil. PO Box 23137 San Juan, Puerto Rico 00931-3137. Tel fijo: (787) 764-0000 ext.86500.jose.serra@upr.edu 


\title{
FATORES PROTETORES DA DEPRESSÁO EM UMA MOSTRA DE ADULTOS MAIORES EM PORTO RICO: AUTO EFICÁCIA, ESCOLARIDADE E OUTRAS VARIÁVEIS SÓCIO DEMOGRÁFICAS
}

\author{
Resumo
}

\begin{abstract}
O objetivo desta pesquisa descritiva-correlacional foi avaliar a relação entre auto eficácia, escolaridade e síntomas depressivos para identificar fatores protetores da depressão em uma mostra de pessoas de idade avançada da população de Porto Rico. Além disso, foram examinadas outras variáveis sócio demográficas como o estado civil, com quem residem e o sexo, com relação à auto eficácia e os sintomas depressivos. Selecionaram-se por disponibilidade 45 participantes com idades entre 60 e 86 anos (M=69; $\mathrm{DE}=7.37)$. Os instrumentos utilizados foram a Escala Auto-avaliativa da Depressão de Zung (1965) e a Escala de Auto eficácia Geral de Baessler e Schwarcer (1996). A hipótese propôs que, a maior auto eficácia e escolaridade, menor seria o nível dos sintomas depressivos em uma mostra de adultos maiores de Porto Rico. As análises estatísticas incluíram testes te os Coeficientes de Correlação de Pearson e de Regressão Múltipla. Foram encontradas diferenças estatisticamente significativas para a auto eficácia e a escolaridade entre os que não apresentavam depressão e os que apresentavam depressão moderada ou severa. Além disso encontraram-se maiores níveis de depressão e menores níveis de auto eficácia nos que haviam enviuvado. Os resultados sugerem que altos níveis de escolaridade e auto eficácia são fatores protetores da depressão nos adultos maiores, enquanto que a viuvez é um fator de risco para depressão.

Palavras chave: depressão, auto eficácia, escolaridade, adultos maiores
\end{abstract}

\section{INTRODUCCIÓN}

A medida que las personas avanzan en edad, ocurren cambios a nivel biológico, psicológico y social. En la vejez suceden eventos vitales que pueden afectar el bienestar de la persona de edad avanzada, como cambio de residencia, retiro o jubilación y la muerte de la pareja y otros seres queridos. Estos cambios suelen implicar un mayor aislamiento social y una disminución de la energía, la movilidad y las funciones sensorio-motoras (Vázquez \& Lozoya, 1994). A su vez, las personas pueden tener actitudes negativas hacia el envejecimiento, debido a la construcción social de la vejez como una etapa de sufrimiento, soledad y pérdidas (Cerquera Córdoba \& Meléndez Merchán, 2010). A nivel biológico, las personas mayores presentan más enfermedades y cambios en la absorción de medicamentos que pueden dificultar los tratamientos (Peña-Solano, Herazo-Dilson, \& Calvo-Gómez, 2009).

Varios investigadores han destacado que los síntomas somáticos y los sentimientos de inutilidad, el pesimismo, la fatiga, pérdida de interés y las alteraciones del sueño son frecuentes en los adultos mayores (Riquelme Marín, Buendía Vidal, \& Ruíz Hernández, 2004). Entre los rasgos diferenciales de la depresión en el adulto mayor se destacan episodios depresivos más prolongados y resistentes al tratamiento, una mayor frecuencia de agitación, irritabilidad, ansiedad, somatizaciones, hipocondría, pérdida de apetito, $\mathrm{y}$ una mayor tendencia a la letargia y formas enmascaradas de la depresión (Calvo Piernagorda, 2008; Ferrey, Le Goues, \& Bobes, 1994). Otros rasgos observados han sido insomnio más intenso, disminución de los rendimientos cognitivos y mayor riesgo de suicidio (Alsina \& Rodríguez, 1995; Peña-Solano et al., 2009).

La depresión en los adultos mayores es un problema de salud pública de gran magnitud y uno de los problemas psiquiátricos más comunes en esta población (Fernández Fernández et al., 2006). Sin embargo, los estudios sobre prevalencia de la depresión en los adultos mayores varían en parte por la variedad de edades utilizadas para definir al adulto mayor. Además, ha habido diversidad de participantes como residenciados, ingresados y ambulatorios, y variabilidad de instrumentos utilizados y estudios según los diferentes subtipos de diagnóstico de depresión (Calvo Piernagorda, 2008).

La prevalencia de depresión en los asilos de ancianos es muy elevada, pero existe una gran dispersión de datos -entre el $14 \%$ y el $70 \%$ - debido en parte a la heterogeneidad de los centros (Riquelme Marín et al., 2004). En general, los estudios sobre adultos mayores señalan que la depresión es más frecuente en las mujeres, en quienes viven solos, son solteros o han enviudado, carecen de estudios, padecen patología física, presentan mayor deterioro cognitivo $\mathrm{y}$ estresores psicosociales, tienen peor percepción de su calidad de vida, y peor salud general (Fernández Fernández et al., 2006; Goel, Muzammil, Kumar, Singh, \& Raghav, 2014; Peña-Solano et al., 2009; Sözeri-Varma, 2012; Yaka, Keskinoglu, Ucku, Yener, \& Tunca, 2014; Zeng, North, \& Kent, 2012).

Investigaciones en Puerto Rico han demostrado que la depresión es la cuarta enfermedad prevaleciente en la población de adultos mayores con un $19.7 \%$, antecedida por la diabetes, la artritis y la presión arterial alta (Palloni, 
Sánchez-Ayéndez, \& Dávila, 2003). Se ha estimado que los síntomas depresivos son más frecuentes en las mujeres, y que el $15 \%$ de la población puertorriqueña sobre los 65 años de edad presenta síntomas depresivos, mientras que la prevalencia de depresión en la población general es menor, con un 4.3\% (Colón de Martí, Guzmán Yunqué, \& Guevara-Ramos, 1997). Algunos estudios realizados en Puerto Rico han encontrado hasta un $20.0 \%$ de sintomatología depresiva en personas mayores de 65 años (Martínez, Guiot, Casas-Dolz, González-Tejera, \& Colón De Martí, 2003), mientras que otros han documentado el diagnóstico de depresión en el $26.9 \%$ de una muestra de personas entre 60 y 64 años de edad (Palloni et al., 2003). Los estudios han encontrado principalmente los siguientes síntomas: retardación psicomotora, confusión, anhedonia y problemas para dormir (Colón de Martí et al., 1997; Martínez et al., 2003).

Es necesario considerar que existe una elevada depresión sub-sindromática en los adultos mayores, ya que la literatura ha resaltado la importancia de la comorbilidad, el deterioro cognitivo y los acontecimientos vitales asociados al envejecimiento, así como otras problemáticas que dificultan el diagnóstico de la depresión en la vejez. Vázquez y Lozoya (1994) afirman que muchos de los síntomas típicos de la depresión son compatibles con los cambios naturales que aparecen con la edad. No obstante, el confundir síntomas o el presumir que los síntomas son ocasionados por la vejez puede ser problemático ya que muchos adultos mayores pueden no estar recibiendo el diagnóstico y tratamiento adecuados.

Menos de la mitad de las personas de edad avanzada con trastorno de depresión mayor en centros de cuidado son diagnosticadas y tratadas (Gellis, McGinty, Horowitz, Bruce, \& Misener, 2007). Riquelme Marín et al. (2004) hallaron que al $71 \%$ de los ancianos deprimidos no se les identifica esta condición por parte de su médico y sólo del 3\% al 14\% reciben algún tipo de tratamiento. Estas dificultades para el diagnóstico de la depresión en los adultos mayores con frecuencia se deben a que los síntomas depresivos que aparecen en estos están íntimamente unidos a otros trastornos físicos y/o psiquiátricos y al uso de medicamentos, lo cual facilita un ocultamiento de los síntomas depresivos y dificulta el diagnóstico (Aktaş et al., 2013; Riquelme Marín et al., 2004). Por otra parte, se ha observado que las personas que trabajan en los hogares de cuidado frecuentemente no reconocen la depresión en los adultos mayores (Brown, Kaiser, \& Gellis, 2007). Un estudio encontró que, al evaluar síntomas clásicos de depresión, los/las participantes atribuyeron los síntomas a depresión si quien los sufría era una persona joven, mientras que cuando fueron presentados por una persona de edad avanzada, los/las participantes atribuyeron los síntomas depresivos a la edad (Ruppel, Jenkins, Griffin, \& Kizer, 2010). Estos estereotipos contribuyen a que la depresión en las personas de edad avanzada no sea diagnosticada y a que estas personas no reciban tratamiento.

Al mismo tiempo, la literatura científica ha sido consistente al señalar que la depresión en el adulto mayor empobrece su calidad de vida y disminuye su expectativa de vida, por lo que es importante reconocerla a tiempo. Esto implica que un diagnóstico adecuado y temprano es importante porque permite la implantación de un tratamiento, al cual pueden responder bien aunque tome más tiempo (Ferrey et al., 1994; Peña-Solano et al., 2009). Por ello, es importante conocer no sólo los factores de riesgo, sino también los factores protectores contra la depresión.

Varios estudios sugieren que la autoeficacia ejerce una influencia poderosa en las personas y la han identificado como un predictor y reductor significativo de síntomas depresivos (Gonyea, O'Connor, Carruth, \& Boyle, 2005; Lin, Liu, \& Jang, 2014; Weng, Dai, Huang, \& Chiang, 2008). La autoeficacia se refiere a las creencias y a la percepción del individuo sobre sus capacidades, con base en lo cual organiza y ejecuta sus actos (Bandura, 1997, 2004). La teoría de autoeficacia de Bandura afirma que aquellos individuos con mayor autoeficacia tendrán más probabilidad de iniciar y persistir en una actividad. Las valoraciones adecuadas de la autoeficacia permiten a la persona no solo ejecutar sus actos, sino también establecer relaciones sociales saludables. Los hallazgos de estudios apoyan esto al observar que los ancianos con altas expectativas de autoeficacia participan con mayor intensidad en actividades físicas y recreativas (Feldberg \& Stefani, 2007; Resnick \& Nigg, 2003).

Cuando las personas con escasa autoeficacia enfrentan situaciones difíciles, sus creencias y pensamientos pesimistas intervienen generando temor, sensaciones de estrés, ansiedad y síntomas depresivos, mientras que las personas que creen poder manejar situaciones difíciles, no se mantienen pensando en estas situaciones de forma pesimista y sienten menos temor (Ribeiro \& Santos, 2015; Visschedijk, van Balen, Hertogh, \& Achterberg, 2013; Zeng et al., 2012). Las creencias de autoeficacia pueden transformar cognitivamente las situaciones que parecen ser amenazantes en situaciones manejables (Ribeiro \& Santos, 2015; van Jaarsveld, Ranchor, Sanderman, Ormel, \& Kempen, 2005). Sin embargo, los patrones de pensamiento negativo, factor relacionado con la depresión, trabajan en sentido contrario al reducir la autoeficacia (Zeng et al., 2012). Como consecuencia, las personas construyen o interpretan sus circunstancias de forma negativa aun cuando existen interpretaciones positivas, lo cual puede, a su vez, conducir a síntomas depresivos (Zeng et al., 2012). De hecho, los 
pacientes que tienen una autoeficacia más alta, presentan menos síntomas depresivos y un mejor ajuste (Gonyea et al., 2005; van Jaarsveld et al., 2005; Weng et al., 2008).

La percepción de los estados emocionales y fisiológicos también influye en la valoración de la autoeficacia. En las actividades que implican fuerza y persistencia, las personas juzgan sus reacciones de estrés, fatiga, dolores y molestias como señales de debilidad física (Ewart, 1992). Asimismo, el estado de ánimo es importante ya que un estado de ánimo positivo fomenta una mayor autoeficacia (Kanavagh \& Bower, 1985).

Por otra parte, existen otras variables que podrían estar relacionadas con la autoeficacia y los síntomas depresivos como son la escolaridad, el estado civil, y el estatus de convivencia o con quien vive la persona (solo/a, con esposo/a, o con familiares). Algunos estudios han encontrado que las personas que poseen un nivel de escolaridad más alto tienen más autoeficacia (Campos \& Pérez, 2007; Hoogendijk et al., 2014) y menos síntomas depresivos (Aktaş et al., 2013; Cañongo-León, Acosta-Castillo, Sosa-Ortiz, \& Prince, 2011; Estrada et al., 2013; Şahin Onat, Ünsal Delialioğlu, \& Uçar, 2014; Teixeira, Vasconcelos-Raposo, Fernandes, \& Brustad, 2013; Yaka et al., 2014). Mientras que el vivir solo o sin pareja, así como el haber enviudado han sido asociados a síntomas depresivos más altos (Cerquera Córdoba \& Meléndez Merchán, 2010; Chan, Malhotra, Malhotra, \& Ostbye, 2011; Goel et al., 2014; Schaan, 2013).

La literatura consultada resalta la influencia significativa de las creencias de eficacia en los síntomas depresivos. Sin embargo, a pesar de que la depresión es la cuarta enfermedad prevaleciente en la población de adultos mayores puertorriqueños, no se ha estudiado el rol de las creencias de autoeficacia y de algunas variables socio-demográficas como factores protectores para reducir la incidencia de síntomas depresivos en esta población. Por ello, el objetivo de esta investigación fue evaluar la relación entre autoeficacia, la escolaridad y los síntomas depresivos para identificar factores protectores de la depresión en una muestra de personas de edad avanzada de la población de Puerto Rico. Como objetivo secundario, se examinaron otras variables sociodemográficas como el estado civil, con quién residen y el sexo en relación a la autoeficacia y los síntomas depresivos. La hipótesis de esta investigación planteó que, a mayor autoeficacia y escolaridad, menor sería el nivel de los síntomas depresivos o deterioro en el estado de ánimo en una muestra de adultos mayores de Puerto Rico.

\section{MÉTODO}

\section{Participantes}

Se seleccionaron por disponibilidad 45 participantes cuyas edades fluctuaban entre los 60 y los 86 años, con una edad promedio de 69 años. Treinta participantes (66.7\%) eran mujeres y 15 (33.3\%) hombres. Los/las participantes no eran residentes del área metropolitana de San Juan, Puerto Rico. El 57.8\% residían en zona rural y $42.2 \%$ en zona urbana.

\section{Instrumentos}

Se construyó una planilla para recoger datos sociodemográficos. Se utilizaron dos instrumentos estandarizados para medir depresión y autoeficacia: la Escala Autoevaluativa de la Depresión de Zung (1965) y la Escala de Autoeficacia General (Baessler \& Schwarcer, 1996; Sanjuán Suárez, Pérez García, \& Bermúdez Moreno, 2000).

La Escala Autoevaluativa de la Depresión de Zung (1965), es una escala auto-administrada que consta de 20 premisas relacionadas con la depresión, de las cuales la mitad está formulada en términos positivos y la otra mitad en términos negativos. La persona cuantifica la frecuencia de los síntomas, no la intensidad, utilizando una escala Likert de 4 puntos, que va desde 1 (raramente o nunca) hasta 4 (casi todo el tiempo o siempre). Las propiedades psicométricas indican que su índice de confiabilidad alfa $(\alpha)$ de Cronbach fluctúa entre 0.79 y 0.92 ; su validez y confiabilidad es similar para personas de edad avanzada y personas adultas más jóvenes, y comparable con instrumentos como el Inventario de Depresión de Beck (Kok, 1994). La Escala Autoevaluativa de la Depresión de Zung ha sido validada para poblaciones latinas (Campo-Arias, Díaz-Martínez, Rueda-Jaimes, Cadena, \& Hernández, 2006) y utilizada para medir síntomas depresivos en colombianos y puertorriqueños (Aldana Olarte \& Pedraza Marín, 2012; Colón de Martí et al., 1997; Martínez et al., 2003; Torres \& Posada, 1993). Este instrumento se caracteriza por ser de fácil administración y altamente sensible para identificar síntomas de depresión (Martínez et al., 2003). Para la interpretación de los resultados se utilizaron los siguientes criterios: menos de 50 puntos, sin depresión; entre 50 y 59, con síntomas leves; entre 60 y 69, con síntomas moderados; y mayor de 69, con síntomas severos. Estos puntos de corte han sido aplicados anteriormente a la población puertorriqueña (Martínez et al., 2003). 
La Escala de Autoeficacia General (Baessler \& Schwarcer, 1996; Sanjuán Suárez et al., 2000), consta de 10 ítems con una escala Likert de 4 opciones $(1=$ incorrecto, $2=$ apenas cierto, $3=$ más bien cierto, $4=$ cierto). Las puntuaciones oscilan entre 10 y 40 puntos. El coeficiente de consistencia interna alfa $(\alpha)$ de Cronbach es .81.

\section{Procedimiento}

Esta investigación utilizó un diseño transversal de una celda y comprendió los siguientes pasos: primero, los/las participantes fueron visitados en sus casas; segundo, a cada participante se le leyó el consentimiento informado y se le solicitó que firmara el mismo, en caso de que quisiera participar en la investigación; tercero, a aquéllos que firmaron, se les entregaron los instrumentos de recopilación de datos; cuarto, se les leyeron los reactivos a quienes tuvieron dificultad para leer y completar los instrumentos; y quinto, los/las participantes entregaron a los investigadores los instrumentos diligenciados en un sobre.

\section{RESULTADOS}

Los datos se analizaron empleando la aplicación SPSS, versión 21.0, y se utilizaron frecuencias relativas y absolutas, media aritmética, pruebas t y los Coeficientes de Correlación de Pearson y de Regresión.

El $51.1 \%$ de los/as participantes estaba casado, $26.7 \%$ viudo, $17.8 \%$ divorciado y el $4.4 \%$ soltero. El $95.6 \%$ tenía algún grado de escolaridad; de este porcentaje, el 46.5\% había completado la primaria; el 30.2\% llegó a la escuela intermedia; el $9.3 \%$ a la escuela superior, y el $14.0 \%$ a la universidad. La mayoría de las mujeres $(58.6 \%)$ había completado estudios primarios, alcanzando en promedio el grado 6, mientras que la mayor parte de los hombres (42.9\%) había completado estudios de escuela intermedia, alcanzando el grado 9 como promedio. Solo un $3.4 \%$ de las mujeres terminó estudios de escuela superior, en comparación con el $21.4 \%$ de los hombres. El $46.5 \%$ residía con sus parejas, el $30.2 \%$ con familiares y el $23.3 \%$ residía solo.

El análisis de la variable Nivel de Síntomas Depresivos, encontró que el 35.6\% presentaba síntomas de depresión leve, $28.9 \%$ tenía problemas de depresión moderada y 4.4\% depresión severa. Los síntomas más frecuentes fueron sentimientos de inutilidad (42.9\%), intranquilidad (38.6\%), dificultad para hacer las cosas $(31.8 \%)$ y dificultad para tomar decisiones (31.7). El 62.5\% de estos participantes tenía una percepción de autoeficacia entre moderada y baja. El promedio de autoeficacia entre los que presentaron depresión moderada o severa fue de $26.7(\mathrm{DE}=8.8)$ y su escolaridad promedio era del grado $6(\mathrm{DE}=4.0)$. De los que no presentaron síntomas depresivos, el $69.4 \%$ obtuvo un nivel alto de autoeficacia con un promedio de 34.2 (DE=6.8). La escolaridad del $42.9 \%$ incluía estudios universitarios y el nivel escolar promedio era el grado $10(\mathrm{DE}=5.5)$. Según se aprecia en la tabla 1, las pruebas t revelaron diferencias estadísticamente significativas en Autoeficacia ( $\mathrm{t}(26)=$ 2.493, $\mathrm{p}=.019$ y Escolaridad t $(25)=2.142, \mathrm{p}=.040$ ) entre los/las participantes que presentaron sintomatología depresiva moderada o severa y los que no presentaron sintomatología depresiva.

La prueba de correlación realizada (véase Tabla 2), reveló que existe una correlación significativa entre la variable Autoeficacia y las variables Escolaridad y Nivel

Tabla 1.

Pruebas t, medias y desviación estándar en escolaridad y autoeficacia de las personas con depresión moderada o severa y las que no presentaron síntomas depresivos.

\begin{tabular}{lccccc} 
& \multicolumn{2}{c}{ Sin Depresión } & \multicolumn{2}{c}{$\begin{array}{c}\text { Con Depresión } \\
\text { Moderada o Severa }\end{array}$} \\
\hline Variables & $\mathrm{M}$ & $\mathrm{DE}$ & $\mathrm{M}$ & $\mathrm{DE}$ & $\mathrm{t}$ \\
\hline Autoeficacia & 34.2 & 6.8 & 26.7 & 8.7 & $2.493^{* *}$ \\
\hline Escolaridad & 9.8 & 5.5 & 5.9 & 4.0 & $2.166^{*}$ \\
\hline
\end{tabular}

Tabla 2.

Correlaciones entre las variables autoeficacia, escolaridad y síntomas depresivos

\begin{tabular}{lcc}
\cline { 2 - 3 } & Escolaridad & Autoeficacia \\
\hline Autoeficacia & $.436^{* *}$ & -- \\
\hline Síntomas Depresivos & $-.359^{*}$ & $-.385^{* *}$ \\
\hline
\end{tabular}

Nota. El estadígrafo utilizado fue la Prueba de Correlación de Pearson $(\mathrm{n}=45)$. El nivel de significancia estadística utilizado fue el siguiente: ${ }^{*} \mathrm{p}<.05 \mathrm{y} * \mathrm{p}<.01$. 
de Síntomas Depresivos, así como una correlación significativa entre las variables Nivel de Síntomas Depresivos y Escolaridad.

El coeficiente de regresión en la prueba de regresión múltiple fue $\mathrm{R}=0.433$ y $\mathrm{R} 2=0.187$ (véase Tabla 3 ). Esto implica que la Autoeficacia y la Escolaridad son variables predictoras y explican el $19.0 \%$ de la varianza de cambio en los Síntomas Depresivos.

Los análisis de otras variables sociodemográficas encontraron diferencias significativas, excepto en el Nivel de Síntomas Depresivos por sexo. Las mujeres presentaron más síntomas depresivos, aunque esta diferencia no fue estadísticamente significativa. Se observó una diferencia significativa por sexo en la variable Autoeficacia donde los hombres obtuvieron un nivel más alto. En cuanto a estatus civil, las personas casadas y las divorciadas presentaron menos síntomas depresivos que las solteras y las viudas, mientras que las casadas obtuvieron el nivel más alto de autoeficacia seguidas por las solteras. Las personas que habían enviudado obtuvieron los peores resultados en ambas variables. No se realizaron análisis por sexo entre quienes enviudaron, ya que el $91.7 \%$ eran féminas. Respecto a con quién residen, se compararon las personas que vivían solas, con su cónyuge, o con familiares. Las que vivían con familiares mostraron los niveles más bajos de autoeficacia, siendo esta diferencia estadísticamente significativa. Aunque también presentaron los niveles más altos de síntomas depresivos, la diferencia no fue significativa. Quienes vivían con su cónyuge obtuvieron los niveles más bajos de síntomas depresivos y los más altos de autoeficacia. No se observaron diferencias por zona de residencia (rural o urbana) en cuanto a autoeficacia y síntomas depresivos.

\section{DISCUSIÓN}

El objetivo principal de esta investigación fue evaluar la autoeficacia, la escolaridad y otras variables sociodemográficas como factores protectores de la depresión en una muestra de adultos mayores de la población de Puerto Rico. Los resultados indican que el $68.9 \%$ de los/las participantes presentaba algún tipo de sintomatología depresiva, donde uno de cada tres adultos mayores (33.3\%) presentó síntomas de depresión moderada o severa. Estos porcentajes son superiores a los reportados en los estudios de Martínez et al. (2003), Colón de Martí et al. (1997), y Palloni et al. (2003). La diferencia en porcentajes se puede deber al tamaño de la muestra, la variabilidad de instrumentos y el lugar de reclutamiento. Se planteó la hipótesis de que a mayor autoeficacia y escolaridad, menor sería el nivel de los síntomas depresivos en la muestra de adultos mayores. Los datos obtenidos indican que se acepta la hipótesis, ya que el Coeficiente de Correlación de Pearson demostró ser significativo en la correlación entre las variables Autoeficacia, Escolaridad y Nivel de Síntomas Depresivos. El 19\% de la varianza en los síntomas depresivos se relacionó con la autoeficacia y escolaridad, lo cual confirma la relevancia de dichas variables protectoras para disminuir la posibilidad de síntomas depresivos en esta muestra.

Las personas con mayor escolaridad demostraron un nivel más alto de autoeficacia y un nivel más bajo de síntomas depresivos. Esto implica que las personas con mayor escolaridad poseen niveles más altos de autoeficacia, lo que reduce la posibilidad de depresión en la muestra. Este hallazgo concurre con los estudios que han encontrado que una mayor escolaridad está asociada a una mayor autoeficacia

Tabla 3.

Análisis de regresión múltiple de la escolaridad y la autoeficacia como factores protectores de los síntomas depresivos

\begin{tabular}{cccc}
\cline { 2 - 4 } & \multicolumn{3}{c}{ Síntomas depresivos } \\
\cline { 2 - 4 } & 6.8 & \multicolumn{2}{c}{ Modelo 2 } \\
\hline Variable & Modelo $1 B$ & $B$ & $95 \%$ IC \\
\hline Constante & $54.634^{* *}$ & $54.474^{* *}$ & {$[45.476,63.472]$} \\
\hline Autoeficacia & $-.362^{*}$ & -.260 & {$[-.570, .050]$} \\
\hline Escolaridad & & -.418 & {$[-.971, .136]$} \\
\hline$R^{2}$ & .140 & .187 & \\
\hline$F$ & $6.675^{*}$ & $4.608^{*}$ \\
\end{tabular}

Nota. $\mathrm{n}=45 . \mathrm{IC}=$ Intervalo de confianza. ${ }^{*} \mathrm{p}<.05 .{ }^{* *} \mathrm{p}<.01$. 
y menores síntomas depresivos (Campos \& Pérez, 2007; Cañongo-León et al., 2011; Hoogendijk et al., 2014; Şahin Onat et al., 2014; Teixeira et al., 2013; Yaka et al., 2014). Ello puntualiza la importancia de fomentar la educación, no solo para un país más productivo, sino también para una futura generación más saludable de adultos mayores.

Los resultados de esta investigación concuerdan con los hallazgos de estudios (Gonyea et al., 2005; Lin et al., 2014; Weng et al., 2008) en los cuales se destaca que la autoeficacia ejerce una influencia poderosa y es un factor predictor en los síntomas depresivos, de manera que, a medida que aumenta la autoeficacia, disminuyen los síntomas depresivos. Algunas investigaciones han destacado que trabajar los procesos cognitivos de las personas mayores y utilizar la revisión de vida, ayuda a disminuir los síntomas depresivos (Moreno-Carrillo \& Lopera-Restrepo, 2010; Serrano-Selva, Latorre Postigo, \& Montañes Rodríguez, 2005). Asimismo, varios estudios han demostrado que es posible aumentar la autoeficacia, y esta a su vez puede transformar cognitivamente las situaciones y mejorar el estado de ánimo, la salud y la calidad de vida (Bandura, 2004; Blazer, 2002; Jones, Mandy, \& Partridge, 2009; Ribeiro \& Santos, 2015; Tse, Vong, \& Tang, 2013; van Jaarsveld et al., 2005). Los pensamientos negativos son un factor vinculado a la depresión y el control sobre estos es una forma en que las creencias de autoeficacia podrían regular la depresión (Zeng et al., 2012). Los resultados sugieren la posibilidad de trabajar con las creencias de autoeficacia de las personas de edad avanzada para generar en ellas una mayor autoeficacia y, de esta manera, protegerse contra la depresión. Será necesario realizar estudios en los que se implementen estrategias para aumentar la autoeficacia y evaluar su efectividad en la población mayor puertorriqueña.

En cuanto a las otras variables sociodemográficas estudiadas, las personas viudas presentaron los niveles más bajos de autoeficacia y los más altos de síntomas depresivos, mientras que las casadas mostraron los niveles más altos de autoeficacia y uno de los más bajos de síntomas depresivos. Esto sugiere que el tener una pareja parece ser un factor protector contra la depresión; no obstante, esta situación cambia al morir la pareja, ya que aumentan los síntomas depresivos, por lo que la viudez es un factor de riesgo para la depresión (Cerquera Córdoba \& Meléndez Merchán, 2010; Goel et al., 2014; Schaan, 2013). Es importante prestar atención a esta condición, ya que la literatura no sólo ha señalado que la muerte de la pareja en una persona de edad avanzada aumenta los síntomas depresivos, sino que a su vez puede afectar adversamente la salud y aumentar la mortalidad de la pareja sobreviviente (Williams Jr., 2005). Por ello resulta necesario desarrollar intervenciones dirigidas a los adultos mayores de manera que puedan prepararse mejor para este evento y afrontarlo más efectivamente.

En relación con el sexo, se observó una diferencia estadísticamente significativa, ya que los hombres presentaron un nivel más alto de autoeficacia que las mujeres. Las mujeres presentaron más síntomas depresivos; no obstante, la diferencia no fue significativa. Este resultado podría estar relacionado con el hecho de que solo un $6.7 \%$ de los hombres había enviudado, en comparación con casi una tercera parte de las mujeres, lo cual puede afectar, tanto la autoeficacia como los síntomas depresivos. Otro factor que podría explicar la diferencia radica en el nivel de escolaridad de los hombres, ya que su escolaridad era más alta que en las mujeres. Por otro lado, es necesario considerar las construcciones sociales relacionadas con el género, las cuales pueden contribuir a que los hombres mayores presenten una mayor autoeficacia y menos síntomas depresivos. En relación con esto, Cerquera Córdoba y Meléndez Merchán (2010) plantean que el varón es conceptualizado como un ser poderoso a quien nada lo puede afectar, por lo que los síntomas se escudan en otras áreas y no se manifiestan como comportamientos depresivos. Una cultura machista que enseña que el hombre no llora, cohíbe la expresión de síntomas depresivos, los cuales pudieran manifestarse de otras maneras, como el alcoholismo. En este entorno sociocultural, el llorar y admitir síntomas relacionados con la depresión es interpretado como debilidad e incluso falta de virilidad, por lo que es menos probable que los hombres se permitan expresar o admitir sentimientos de tristeza. De igual forma, la construcción social del hombre que todo lo puede, hace que sea más probable que los adultos mayores presenten una mayor autoeficacia.

Este estudio encontró que los adultos mayores que vivían con sus familiares presentaban niveles de autoeficacia significativamente más bajos, lo cual podría sugerir que estas personas sienten que no pueden valerse por sí mismas o que son una carga física y económica al depender de sus familiares, lo cual puede generar pensamientos de inutilidad (Estrada et al., 2013; Yaka et al., 2014; Zeng et al., 2012). En relación con esto,,es necesario señalar que el $69.2 \%$ de los/las participantes que vivía con familiares había enviudado, factor que afecta la autoeficacia y la depresión. Por otra parte, más del 50\% tenía 70 años o menos, por lo que es probable que aún manifestara cierta autosuficiencia. Los adultos mayores que tienen mayor apoyo social presentan menos síntomas depresivos (Castellano Fuentes, 2014; Chan et al., 2011; Lino, Portela, Camacho, Atie, \& Lima, 2013), mientras que los que reportan menos relaciones sociales y actividades físicas favoritas presentan más síntomas depresivos (Timalsina, Sherpa, \& Dhakal, 2014), 
por lo que es importante fortalecer los vínculos sociales y proveer actividades de esparcimiento, sobre todo en aquellos que viven solos o han enviudado. Ello no necesariamente significa que lo mejor es que los adultos mayores vivan con sus familiares, ya que en algunos casos pueden sentirse como una carga para éstos, y menos autosuficientes. Es posible que un círculo social activo y visitas frecuentes de familiares sean de mayor beneficio para algunas personas mayores que el vivir con sus familiares, en particular para aquellas que aún son autosuficientes. Esto es algo que debe ser evaluado según las preferencias y necesidades de cada adulto mayor. En el caso de aquellos cuya condición requiere que no vivan solos, es necesario proveerles actividades, atención de calidad y participación activa en las decisiones, a la vez que se respeta su espacio, de manera que sientan que su opinión y sus sentimientos tienen valor y son importantes.

Entre las intervenciones que se podrían emplear con los adultos mayores se encuentran el implementar programas dirigidos al aumento en la autoeficacia a través de la identificación de estrategias para afrontar los obstáculos más efectivamente, como por ejemplo, el compensar limitaciones causadas por enfermedades físicas al aprovechar fortalezas alternas (Blazer, 2002). Si junto con estrategias a nivel social y biológico se enseñan habilidades para aumentar la autoeficacia y controlar los pensamientos negativos como complemento de la revisión de vida, será posible ayudar a prevenir e incluso disminuir síntomas depresivos en los adultos mayores.

Este estudio identificó la viudez como un factor de riesgo para la depresión, por lo que es vital que quienes han enviudado reciban apoyo a nivel familiar, social e institucional durante este difícil proceso. Es necesario que haya un monitoreo para evaluar si la persona sobreviviente ha desarrollado depresión y necesita psicoterapia o farmacoterapia para ayudarle a lidiar con este evento. El apoyo y la orientación a los familiares es importante para que ayuden a la persona sobreviviente sin que esta se sienta inútil y para que sepan reconocer cuándo la persona sobreviviente necesita otras ayudas. Las políticas públicas deben asegurar que las personas mayores tengan la oportunidad de participar de actividades sociales que promuevan el esparcimiento y la confraternización. Asimismo, deben promover el que los centros de cuidado de adultos mayores provean actividades que les ayuden a mantener activos su mente y su cuerpo, a través de ejercicios físicos y juegos; e igualmente estimular el disfrute de los sentidos utilizando la música y otras expresiones de arte, los aromas y sabores.

$\mathrm{Al}$ trabajar por el bienestar del adulto mayor, debemos pensar no solamente en la población mayor actual, sino también en las futuras. La política pública debe asegurar que a través de la educación se fomente la autosuficiencia independientemente del sexo de las personas, por ejemplo, asegurándose de que tanto hombres como mujeres aprendan a cocinar, y que se promueva el que ambos participen de las tareas de limpieza del hogar y de asuntos como el manejo del dinero y la toma de decisiones. Ello facilita el que tanto hombres como mujeres participen de manera equitativa en el hogar y que puedan ser autosuficientes en caso de perder a su pareja. La implementación de este tipo de educación puede ser un gran reto en las culturas latinas, pero no imposible. Asimismo, es importante que se atienda la deserción escolar y que las políticas públicas fomenten la equidad en la oportunidad de empleo e ingresos para una futura generación de adultos mayores que se sienta más realizada.

Una limitación de este estudio está relacionada con el hecho de que, aunque el instrumento utilizado ha sido traducido y validado para identificar síntomas depresivos en la población colombiana (Campo et al., 2006), se ha sugerido que pueden existir diferencias culturales con la población puertorriqueña en la interpretación de los enunciados (Martínez et al., 2003). Otra limitación está vinculada al pequeño tamaño de la muestra, por lo cual se sugiere realizar estudios con muestras más grandes. A pesar de las limitaciones metodológicas, este trabajo constituye una importante aportación, ya que en Puerto Rico se han realizado pocas investigaciones sobre los factores relacionados con la depresión en los adultos mayores. Puntualizamos la importancia de prestar atención a esta población que presenta un alto índice de suicidios (Alsina \& Rodríguez, 1995; Peña-Solano et al., 2009) y al hecho de que muchos adultos mayores deprimidos no reciben diagnóstico ni tratamiento para la depresión (Brown et al., 2007; Gellis et al., 2007; Riquelme Marín et al., 2004). Existe la necesidad de más estudios epidemiológicos y psiquiátricos con la población de edad avanzada puertorriqueña, así como intervenciones que consideren y trabajen de manera integral los aspectos cognitivos, sociales y biológicos que inciden en el bienestar de la persona de edad avanzada.

\section{REFERENCIAS}

Aktaş, C., Eren, Ş. H., Korkmaz, İ., Caslişkan, H. M., Karcioğlu, Ö., \& Sarikaya, S. (2013). Depression in the elderly patients admitted to the emergency department: A multicenter study. Turkish Journal of Geriatrics, 16(1), 20-24.

Aldana Olarte, R. \& Pedraza Marín, J. A. (2012). Análisis de la depresión en el adulto mayor en la encuesta nacional de demografía y salud 2010. Recuperado de http://repository. urosario.edu.co/bitstream/10336/3002/3/93206082-2012.pdf

Alsina, S., \& Rodríguez, J. (1995). El suicidio en ancianos puertorriqueños. Revista Latinoamericana de Psicología, 27(2), 263-282. Recuperado de http://www.redalyc.org/ articulo.oa?id $=80527207$ 
Baessler, J., \& Schwarcer, R. (1996). Evaluación de la autoeficacia: Adaptación española de la Escala de Autoeficacia General. Ansiedad y Estrés, 2, 1-8.

Bandura, A. (1997). Self-efficacy: The exercise of control. New York: W. H. Freeman/Times Books/Henry Holt \& Co.

Bandura, A. (2004). Health promotion by social cognitive means. Health Education \& Behavior, 31(2), 143-164. doi: $10.1177 \% 2 \mathrm{~F} 1090198104263660$

Blazer, D. G. (2002). Self-efficacy and depression in late life: A primary prevention proposal. Aging and Mental Health, 6(4), 315-324.

Brown, E. L., Kaiser, R. M., \& Gellis, Z. D. (2007). Screening and assessment of late life depression in home healthcare: Issues and challenges. Annals of Long Term Care, 15(10), 27-32.

Calvo Piernagorda, C. M. (2008). Marcadores lingüisticos y mnésicos en el diagnóstico diferencial de la depresión y la demencia: Un estudio con seguimiento (Disertación doctoral). Recuperado de http://eprints.ucm.es/8292/1/ T30580.pdf

Campo-Arias, A., Díaz-Martínez, L. A., Rueda-Jaimes, G. E., Cadena, L.P., \& Hernández, N. L. (2006). Validation of Zung's self-rating depression scale among the Colombian general population. Social Behavior and Personality, 34(1), 87-94. doi: 10.2224/sbp.2006.34.1.87

Campos, S., \& Pérez, J. C. (2007). Autoeficacia y conflicto decisional frente a la disminución del peso corporal en mujeres. Revista Chilena de Nutrición, 34(3), 213-218. Recuperado de http://www.scielo.cl/scielo.php?pid=S071775182007000300004\&script $=$ sci_arttext

Cañongo-León, G., Acosta-Castillo, I., Sosa-Ortiz, A. L., \& Prince, M. J. (2011). Asociación de síntomas depresivos con variables sociodemográficas y red social. Archivos de Neurociencia, 16(1), 10-13.

Castellano Fuentes, C. L. (2014). La influencia del apoyo social en el estado emocional y las actitudes hacia la vejez y el envejecimiento en una muestra de ancianos. International Journal of Psychology \& Psychological Therapy, 14(3), 365-377.

Cerquera Córdoba, A. M., \& Meléndez Merchán, C. (2010). Factores culturales asociados a las características diferenciales de la depresión a través del envejecimiento. Pensamiento Psicológico, 7(14), 63-71.

Chan, A., Malhotra, C., Malhotra, R., \& Ostbye, T. (2011). Living arrangements, social networks and depressive symptoms among older men and women in Singapore. International Journal of Geriatric Psychiatry, 26(6), 630639. doi: $10.1002 / \mathrm{gps} .2574$

Colón de Martí, L. N., Guzmán Yunqué, F. S., \& GuevaraRamos, L. M. (1997). Early detection of depression using the Zung Self-Rating Depression Scale. Puerto Rico Health Science Journal, 16(4), 375-379.

Estrada, A., Cardona, D., Segura, A. M., Ordóñez, J., Osorio, J. J. \& Chavarriaga, L. M. (2013). Síntomas depresivos en adultos mayores institucionalizados y factores asociados. Universitas Psychologica, 12(1), 81-94.

Ewart, C. K. (1992). Role of physical self-efficacy in recovery from heart attack. En R. Sachwarzer (Ed.), Self-efficacy: Thought control of action. Washington: Hemisphere.

Feldberg, C., \& Stefani, D. (2007). Autoeficacia y rendimiento en memoria episódica verbal, y su influencia en la participación social de las personas de edad. Anales de Psicología, 23(2), 282-288.

Fernández Fernández, C., Cabeller García, J., Saiz Martínez, P. A., García-Portilla González, M. P., Martínez Barrondo, S., \& Bobes García, J. (2006). La depresión en el anciano en una zona rural y su interacción con otros factores. Actas Españolas de Psiquiatría, 34(6), 355-361.

Ferrey, G., Le Goues, G., \& Bobes, J. (1994). Psicopatología del anciano. Barcelona: Masson.

Gellis, Z. D., McGinty, J., Horowitz, A., Bruce, M., \& Misener, E. (2007). Problem solving therapy for late life depression in home care: A randomized field trial. American Journal of Geriatric Psychiatry, 15(11), 968-978. doi: 10.1097\%2FJGP.0b013e3180cc2bd7

Goel, P. K., Muzammil, K., Kumar, S., Singh, J. V., \& Raghav, S. K. (2014). Socio-demographic correlates of depression among elderly slum dwellers of North India. Nepal Journal of Epidemiology, 4(1), 316-322.

Gonyea, J. G., O'Connor, M., Carruth, A., \& Boyle, P. A. (2005). Subjective appraisal of Alzheimer's disease caregiving: the role of self-efficacy and depressive symptoms in the experience of burden. American Journal of Alzheimer's Disease \& Other Dementias, 20(5), 273-280. doi:10.1177 $\% 2 \mathrm{~F} 153331750502000505$

Hoogendijk, E. O., van Hout, H. P.J., Heymans, M. W.; van der Horst, H. E., Frijters, D. H. M., Broese van Groenou, M. I., Huisman, M. (2014). Explaining the association between educational level and frailty in older adults: results from a 13-year longitudinal study in the Netherlands. Annals of Epidemiology, 24(7), 538-544.e2. 0p. doi: 10.1016/j. annepidem.2014.05.002

Jones, F., Mandy, A., \& Partridge, C. (2009). Changing selfefficacy in individuals following a first time stroke: preliminary study of a novel self-management intervention. Clinical Rehabilitation, 23(6), 522-533.

Kanavagh, D. J., \& Bower, G. H. (1985). Mood and selfefficacy: Impact of joy and sadness on perceived capabilities. Cognitive Therapy and Research, 9(5), 507525. doi: 10.1007\%2FBF01173005

Kok, R. M. (1994). Self-evaluation scales for depression in the elderly. Tijdschrift Gerontologie Geriatrie, 25(4), 150-156.

Lin, S., Liu, J., \& Jang, Y. (2014). The role of psychosocial and cultural factors as correlates of depressive symptoms in Chinese-American older adults. Clinical Gerontologist, 37(2), 108-119. doi: 10.1080/07317115.2013.868847

Lino, V., Portela, M., Camacho, L., Atie, S., \& Lima, M. (2013). Assessment of social support and its association to 
depression, self-perceived health and chronic diseases in elderly individuals residing in an area of poverty and social vulnerability in Rio de Janeiro City, Brazil. PLOS ONE, $8(8), 1-7$.

Martínez, K. G. Guiot, H. M., Casas-Dolz, I., González-Tejera, G., \& Colón De Martí, L. N. (2003). Applicability of the Spanish Translation of the Zung Self-Rating Depression Scale in a general Puerto Rican population. Puerto Rico Health Sciences Journal, 22(2), 179-85.

Moreno-Carrillo, C., \& Lopera-Restrepo, F. (2010). Efectos de un entrenamiento cognitivo sobre el estado de ánimo. Revista Chilena de Neuropsicología, 5(2), 146-152.

Palloni, A., Sánchez-Ayéndez, M., \& Dávila, A. L. (2003). La Salud de los Adultos de Edad Mayor en Puerto Rico. Recuperado de http://prehco.rcm.upr.edu/docs/Inform1.pdf

Peña-Solano, D. M., Herazo-Dilson, M. I., \& Calvo-Gómez, J. M. (2009). Depresión en ancianos. Revista Facultad de Medicina de la Universidad Nacional de Colombia, 57(4), 347-355.

Resnick, B., \& Nigg, C. (2003). Testing a theoretical model of exercise behavior for older adults. Nursing Research, 52(2), 80-89.

Ribeiro, O., \& Santos, A.R. (2015). Psychological correlates of fear of falling in the elderly. Educational Gerontology, 41(1), 69-78. doi: 10.1080/03601277.2014.924272

Riquelme Marín, A., Buendía Vidal, J., \& Ruíz Hernández, J.A. (2004). Estructura diferencial de la depresión en ancianos. Psiquis, 25(3), 108-121.

Ruppel, S.E., Jenkins, W. J., Griffin, J. L., \& Kizer, J. B. (2010). Are they depressed or just old? A study of perceptions about the elderly suffering from depression. North American Journal of Psychology, 12(1), 31-42.

Şahin Onat, S., Ünsal Delialioğlu, S., \& Uçar, D. (2014). The risk of depression in elderly individuals, the factors which related to depression, the effect of depression to functional activity and quality of life. Turkish Journal of Geriatrics, 17(1), 35-43.

Sanjuán Suárez, P., Pérez García, A. M., \& Bermúdez Moreno, J. (2000). Escala de Autoeficacia General: Datos Psicométricos de la Adaptación para Población Española. Psicothema, 12(2), 509-513.

Schaan, B. (2013). Widowhood and depression among older Europeans - The role of gender, caregiving, marital quality, and regional context. Journals of Gerontology, Series B: Psychological Sciences and Social Sciences, 68(3), 431-442, doi:10.1093/geronb/gbt015

Serrano-Selva, J. P., Latorre Postigo, J. M., \& Montañes Rodríguez, J. (2005). Terapia sobre revisión de vida basada en la recuperación de recuerdos autobiográficos específicos en ancianos que presentan síntomas depresivos. Revista Española de Geriatría y Gerontología, 40(4), 228-235.

Sözeri-Varma, G. (2012). Depression in the elderly: Clinical features and risk factors. Aging and Disease, 3(6), 465-471.
Teixeira, C., Vasconcelos-Raposo, J., Fernandes, H., \& Brustad, R. (2013). Physical activity, depression and anxiety among the elderly. Social Indicators Research, 113(1), 307-318. doi: 10.1007/s11205-012-0094-9

Timalsina, R., Sherpa, P. D., \& Dhakal, D. K. (2014). Factors associated with depression among elderly living in old age homes in Kathmandu Valley. Journal of Institute of Medicine, 36(1), 90-96.

Torres, Y., \& Posada, J. (1993). Estudio nacional de salud mental y consumo de sustancias psicoactivas. Bogotá: Ministerio de Salud.

Tse, M. M., Vong, S. K., \& Tang, S. K. (2013). Motivational interviewing and exercise programme for communitydwelling older persons with chronic pain: A randomized controlled study. Journal of Clinical Nursing, 22(13/14), 1843-1856. doi: 10.1111/j.1365-2702.2012.04317.x

van Jaarsveld, C. H. M., Ranchor, A. V., Sanderman, R., Ormel, J., \& Kempen, G. I. J. M. (2005). The role of premorbid psychological attributes in short- and long-term adjustment after cardiac disease. A prospective study in the elderly in The Netherlands. Social Science \& Medicine, 60(5), $1035-$ 1045. doi: 10.1016/j.socscimed.2004.06.037

Vázquez, C., \& Lozoya, G. (1994). Evaluación y diagnóstico diferencial de los trastornos depresivos en personas de edad. En J. Buendía (comp.), Envejecimiento y psicología de la salud. Madrid: Siglo Veintiuno.

Visschedijk, J., van Balen, R., Hertogh, C., Achterberg, W. (2013). Fear of falling in patients with hip fractures: Prevalence and related psychological factors. Journal of the American Medical Directors Association, 14(3), 218-220 doi: 10.1016/j.jamda.2012.10.013

Weng, L. C., Dai, Y. W., Huang, H. L., \& Chiang, Y. J. (2008). Effects of self-efficacy, self-care behaviours on depressive symptom of Taiwanese kidney transplant recipients. Journal of Clinical Nursing, 17, 1786-1794. doi: 10.1111\%2Fj.13652702.2007.02035.x

Williams Jr., J. R. (2005). Depression as a mediator between spousal bereavement and mortality from cardiovascular disease: Appreciating and managing the adverse health consequences of depression in an elderly surviving spouse. Southern Medical Journal, 98(1), 90-95. doi: 10.1097\%2F01.SMJ.0000140832.14297.61

Yaka, E., Keskinoglu, P., Ucku, R., Yener, G. G., \& Tunca, Z. (2014). Prevalence and risk factors of depression among community dwelling elderly. Archives of Gerontology \& Geriatrics, 59(1), 150-154. doi: 10.1016/j. archger.2014.03.014

Zeng, W., North, N., \& Kent, B. (2012). A framework to understand depression among older persons. Journal of Clinical Nursing, 21(17-18), 2399-2409. doi: 10.1111/j.1365-2702.2011.04049.x

Zung, W. W. K. (1965). A self-rating depression scale. Archives of General Psychiatry, 12(1), 63-70. 